\title{
The functional neuro-anatomy of the human response to fear: A brief review
}

\author{
A Del Casale, MD \\ $S$ Ferracuti, MD \\ G D Kotzalidis, MD \\ C Rapinesi, MD \\ D Serata, MD \\ E Ambrosi, MD \\ A Simonetti, MD \\ G Serra, MD \\ V Savoja, MD
}

Sapienza University and School of Medicine and Psychology, NESMOS Department (Neurosciences, Mental Health, and Sensory Functions), Sant'Andrea Hospital, Rome, Italy

M Pompili, MD, PhD

Sapienza University and School of Medicine and Psychology, NESMOS Department, Sant'Andrea Hospital, and Harvard Medical School, McLean Hospital, Belmont, MA, USA

\section{G Angeletti, MD \\ R Tatarelli, MD \\ P Girardi, MD}

Sapienza University and School of Medicine and Psychology, NESMOS Department, Sant'Andrea Hospital

Fear perception and subsequent appropriate behavioural response are crucial for the adaptation of species. During the past few decades functional neuro-imaging studies in humans exploring the neural basis of fear have contributed significantly to the understanding of its mechanisms. Imaging studies help to clarify the role of the amygdala-based neurocircuitry in fear activation. The aim of this paper is briefly to review the most recent functional neuro-imaging studies on fear perception, modulation and learning.

Important knowledge has been acquired about the factors that set fear in motion, including the role of nonconscious processes and how fear drives attention. A subcortical network interacts with the prefrontal cortex to modulate emotional responses that allow better coping with environmental and social demands. Fear learning reduces the need to relearn about dangerous stimuli. Flexible processes allow fear behaviour to be readjusted when circumstances change. Future improvement of functional and other neuro-imaging techniques may better clarify the role of the neurocircuitry involved in fear perception, learning and modulation

Being exposed to fear signals makes us feel threatened and prompts us to prepare an adaptive response. Fear perception and appropriate behavioural responses are crucial for environmental adaptation and survival of species. Sympathetic activation after fear perception triggers the fight or flight (or freeze) reaction, which allows an attempt to meet the stressful situation in an adaptive manner. Furthermore, the transmission or sharing of fear within social groups may represent a defensive mechanism for many species

The neurobiology of the detection of threats and fear has long been studied using animal models. Sensory input signalling danger may gain rapid access to the amygdala through both bottom-up and top-down mechanisms, involving the thalamus and sensory cortices, respectively. The subcortical pathway activating the amygdala passes through the superior colliculi and the pulvinar of the thalamus before accessing it. This pathway operates at low spatial frequency information.

The amygdala appears to play a role in both threat assessment and fear conditioning, as well as in other associative forms of learning, such as responding to potentially threatening stimuli. ${ }^{2,3}$ It also mediates significant autonomic responses to threat through its descending projections to brainstem nuclei. ${ }^{4.5}$ Furthermore, its projections to several layers of the visual cortex probably support sensory processing modulation. ${ }^{\circ}$ The response of the amygdala to threatening stimuli is probably modulated by a network involving the infralimbic prefrontal cortex, which inhibits the activation of the amygdala, ${ }^{7.8}$ and the hippocampus, which is involved in learning about safe versus dangerous contexts. It has been speculated that similar brain mechanisms may underlie contextual fear conditioning across species. ${ }^{10}$

Modern neuro-imaging techniques have significantly fostered the identification of anatomical structures and networks involved in fear perception and response. The aim of this paper is briefly to review recent literature on functional imaging that focuses on the detection of threat and human fear.

\section{Method}

We searched Medline and Psyclnfo databases using the terms 'fear', 'fear response', 'fear conditioning', 'fear extinction', 'threat detection', 'magnetic resonance imaging', 'functional', 'positron emission tomography' and 'single photon emission computerized 
tomography'. More papers, which did not appear in this search, were found in the reference lists of retrieved papers. Papers were considered for inclusion only if they were published in peerreviewed journals.

Papers were included if they satisfied standards for adequate methodology and inclusion criteria. Studies with inadequate methodology /method unspecified and/or inadequately described) were excluded. We also excluded papers that did not include a healthy control group in their analyses. Most of these studies were published in the last 10 years.

\section{Fear perception and modulation}

Several functional imaging studies focused on areas activated in healthy people after fearful stimuli. The basic forms of associative learning, i.e. fear conditioning and extinction, are becoming increasingly important paradigms. They have pathophysiological and therapeutic implications, since they increase our understanding of the anxiety disorders, facilitate their appropriate classification, and allow us to gauge the effects of their treatment," both pharmacological and psychotherapeutic. These forms of learning have been exploited by creating paradigms of conditioning/ extinction resulting in a reduction of fear; this has been used in functional neuro-imaging studies focusing on the shift from fear to safety. $^{12}$

\section{Fear perception}

Functional imaging studies showed increased activation of the amygdalae after fear stimuli in healthy volunteers. ${ }^{13,14}$ Garrett and Maddock, ${ }^{15}$ using functional magnetic resonance imaging (fMRI) with a fear-provocation paradigm, showed that the amygdala, rather than responding to a specifically defined fear stimulus, is set to initially respond to a general threat potential of the stimulus in question. Following conscious perception of stimuli evoking a real fear condition, there is strong bilateral amygdala activation. ${ }^{13,14}$ This mechanism seems to operate similarly in healthy women and men. Given its function-detection properties, an intact amygdala is needed for salient stimulus processing. ${ }^{16}$ The perception of fear elicits specific activity in the right temporoparietal junction," probably representing an attempt to prepare and process an adequate adaptive response.

\section{Modulation and top-down inhibition of fear}

When a coping strategy seems not to be directly available ${ }^{18}$ and fear-related emotions still persist after stimulus perception, increased activation in both the amygdala and the dorsomedial/ dorsolateral prefrontal cortex constitutes a measure of the ongoing emotional conflict. This mechanism seems to be active in specific phobia and other psychiatric disorders. If stimuli evoke a nonfeared condition, or a condition that can be coped with, the activity in the amygdala is reduced. 15,19,20 In such instances, there is an inhibitory modulation principally from the prefrontal cortex, ${ }^{2123}$ the orbitofrontal cortex $x^{24}$ and the anterior cingulate cortex (topdown inhibition). ${ }^{725,26}$ It has been shown that effective connectivity between the amygdala and orbitofrontal cortex allows for more precise perception of facial expressions and differentiated characterisation of emotional qualities. ${ }^{27}$ In this study, Liang et al. ${ }^{27}$ found greater effective connectivity, from the lateral orbitofrontal cortex to the amygdala than from the medial orbitofrontal cortex to the amygdala during fear perception, compared with neutral, positive and other negative emotions. Effective connectivity is the influence that one neural system exerts over another at any level, synaptic or neuronal group. Ward et al. ${ }^{28}$ proposed a role for the pulvinar in the recognition of fearful expression. They considered both direct and indirect cortical connections of the pulvinar with the amygdala, and suggested a primary integrative role for the former. The medial amygdala receives innervation from other areas and projects to many areas, and also constitutes an intermediate relay in neural pathways initiating elsewhere and terminating elsewhere; the amygdala receives input from both medial and lateral sections of the orbitofrontal cortex, which control fear-related processes. ${ }^{14}$ These integrated mechanisms may be affected by neuro-endocrine control. In particular, it was shown that testosterone depresses amygdalar responses to fearful faces, resulting in a prolonged reaction time. ${ }^{10}$

Amygdalar damage may affect emotional experience and expression, but were found not to affect the person's general disposition towards emotions in one study. ${ }^{29}$ Consequently it was hypothesised that the amygdala participates in the perception of emotion-related stimuli, but is not directly involved in the subjective negative emotional experience that persists after the offset of the evoking stimulus.'

Emotional processing is modulated by a network involving the left hippocampus, the right lateral orbitofrontal cortex, the thalamus and the cerebellum. In this network, Brodmann's area (BA) 47 in the lateral orbitofrontal cortex is the most frequently activated prefrontal region in studies focusing on emotion. ${ }^{30}$ BA 47 may be involved in emotional and non-emotional metacognitive selfmonitoring and self-reflection processes, as well as in regulation of the emotions. ${ }^{31}$

Hippocampal activation is probably related to enhanced aversive stimuli memory encoding. ${ }^{15}$ It seems that other brain regions, principally the insular and somatosensory cortices and the parahippocampal gyrus, along with the anterior cingulate and dorsolateral prefrontal cortices, are involved in the anticipation of aversive affective images. ${ }^{32,33}$ A model for top-down inhibition of fear is shown in Fig. 1

\section{The amygdala: response, lateralisation, and modulation}

All emotional stimuli are associated with a higher probability of amygdalar activation than neutral stimuli. Similar effects are observed for most negative and positive emotions, with a higher probability of activation for fear and disgust relative to happiness. The level of attentional processing affects the activity of the 


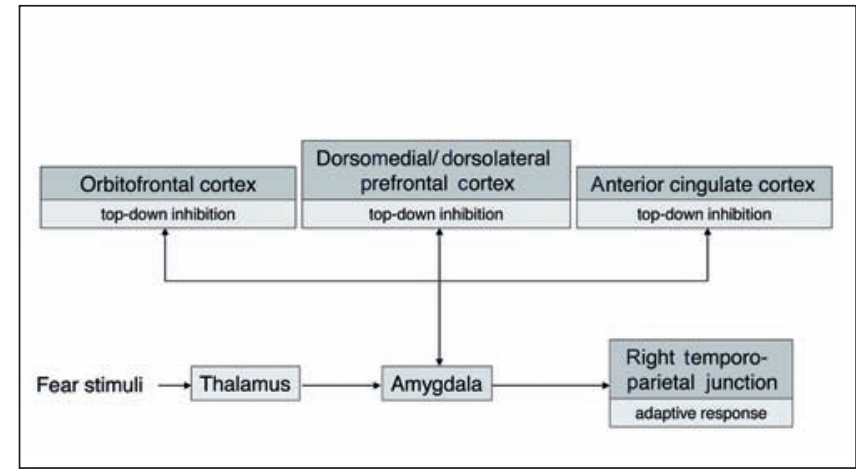

Fig. 1. Primary brain areas involved in top-down inhibition after fear perception.

amygdala; in fact, passive processing has a higher probability of activation compared with active task instructions. The probability of activation of the amygdala increases with aversive learning ${ }^{34}$ and also according to stimulus modality. For example, gustatoryolfactory and visual stimuli increase the probability of activation compared with internal stimuli such as interoceptive stimuli from the gut, heart and the like.

The combination of fearful faces and averted gazes activates areas related to gaze shifting (superior temporal and intraparietal sulci) and fear processing (amygdala, hypothalamus, pallidum). Additional modulation of activation was observed in motion detection areas, in premotor areas and in the somatosensory cortex, bilaterally. It was therefore suggested that gaze direction triggers a process in the brain that takes into account both facial expression and gaze direction when it computes and formulates a final hypothesis on what the other's emotion (fear in this case) is or what the behavioural consequences for the observer will be. ${ }^{35}$

A direct role for the amygdala was found in reflex gaze initiation towards fearfully widened eyes. ${ }^{36}$ Corneal exposure, obtained through moving the eyes laterally or vertically, is a measure of gaze and is also called white eye area. The amount of white in one's exposed eyeball is believed to be a significant cue in activating the amygdala. At similar corneal exposure increases, fearful eyes and gaze shifts in the right-handed are distinguished better by the left than by the right amygdala. Similarly, the left fusiform gyrus was more able than the right in fear discrimination tasks. However, differently from the amygdala, it also responded to all other emotions. Hemispheric differences in white eye area-related fear brain activation suggest that emotional face information is coded by hemispheric-specific mechanisms. In fact, the left (dominant) amygdala proved to be more able than the right in discriminating between fearful eyes and gaze, even at the same levels of white eye area exposure. ${ }^{37}$

Both the thalamus and the ventral portion of the anterior cingulate cortex showed a negative covariation (reciprocal) with the left and right amygdala during fearful face processing, suggesting dynamic interaction between the thalamus, the amygdala, and cortical regions during such processing. ${ }^{38}$
Differential activations to fearful versus neutral faces were observed not only in the amygdala, but also in the pulvinar and in the superior colliculus. These three structures showed a predominance of activation on the left over the right hemisphere only when faces were presented in the left hemifield. ${ }^{39}$ Subcortical left predominance for processing of stimuli rated as dangerous extends also to fear-related behaviours. ${ }^{39}$

Some evidence for hemispheric specialisation, with a relative left-lateralisation for stimuli containing language and a relative right-lateralisation for masked stimuli (stimuli inserted abruptly and unexpectedly to render index stimuli of a task imperceptible or barely perceptible; they may be auditory or visual, simultaneous, pre-stimulus (backward masking) or post-stimulus (forward maskingll, has been reported in a meta-analysis. ${ }^{34}$

These results suggest a leftward bias in subcortical fear processing and highlight the importance of hemifield advantage in emotional lateralisation, which might reflect the combination of hemispheric dominance and asymmetrical interhemispheric information transfer. ${ }^{39}$ Other evidence suggests that the amygdala can use information from facial regions other than the eyes, enabling responses that differentiate fearful from neutral faces, even when the eye region is hidden. ${ }^{40}$

Furthermore, the amygdala is also involved in orientating to backward-masked fearful faces. This spatial attention-related response has been correlated with activity in the anterior cingulate, the superior temporal sulcus and the lingual gyrus. ${ }^{41}$

A role for the human amygdala in the general detection of behaviourally relevant stimuli is supported by the observation that bilateral amygdala activation is stronger in response to behaviourally relevant letter stimuli compared with letters with less behavioural relevance. ${ }^{42}$ Behaviourally relevant letters were letters that people had to distinguish from other letters associated with prepotent responses (habitual learned responses), thus differentiating their response (for example, when people are instructed to move their right hand when viewing any letter but $b$, that should elicit a response with the left hand; the latter is the behaviourally relevant one).

Bilateral amygdala response to fearful faces may be modulated by culture and context, suggesting that both automaticity and topdown regulation are important in emotional regulation. Chiao et al. ${ }^{43}$ used FMRI to measure the response of the amygdala to fearful and non-fearful faces in two different cultures. Native Japanese in Japan and Caucasians in the USA showed greater amygdala activation to fear expressed by members of their own cultural group. This indicates that cultural regulation intervenes top-down to modulate automatic neural responses ${ }^{43}$ dealing with such basic human emotional attitudes as altruistic disposition and empathic attitude. These two emotional attitudes are also regulated by innate, bottom-up processes. ${ }^{44,45}$ Combined, integrated top-down and bottom-up mechanisms for fear perception in the amygdala 
are also supported by findings regarding functional connectivity. It appears that one 'automatic', bottom-up, subcortical-amygdaloid pathway mediates non-consciously perceived fear, whereas consciously perceived fear is mediated by both subcorticalamygdaloid and cortical amygdaloid pathways. ${ }^{46}$

In healthy people these responses differ with age. A study found a significant negative correlation between increasing age and the neural response to fearful and disgusted expressions in the dorsomedial prefrontal cortex (BA 10) and middle frontal gyri (BA 6). ${ }^{47,48}$ Hence, in healthy people the functional anatomy of facial emotion processing is not fixed, but undergoes progressive refinement into adulthood. Age-related changes in dorsomedial and middle frontal cortical activity may be related to some sort of habit formation or 'automatisation'. This results in a decrease in the attention required for appraising facial expressions. Regarding social and cognitive development, it parallels an increase in perceptual skills regarding the ability to assess the relevance of facial expressions. ${ }^{47}$

Frontolimbic structures involved in fear conditioning have been related to an efficient, resilient hypothalamic-pituitary-adrenal (HPA) axis, which interacts with amygdalar, hippocampal and ventromedial prefrontal cortex region activity. The reactivity of cortisol to emotional visual scenes was positively associated with amygdalar and hippocampal activities and negatively with ventromedial prefrontal cortex activity. ${ }^{48}$

In neuro-imaging studies of human fear conditioning, different patterns of brain activation were shown when paired and unpaired conditioned stimuli were presented to healthy right-handed human volunteers. Paired stimuli increased activation in the amygdala, anterior cingulate and fusiform gyrus, whereas unpaired ones activated the insula and left dorsolateral prefrontal cortex. ${ }^{19}$ Unconditioned stimulus response (UCR) decreases when the stimulus may be predicted, a phenomenon called UCR diminution. For unconditioned, aversive, fearful stimuli, when expectancy of the unconditioned fearful stimulus increases by pairing it with a conditioned stimulus, UCR diminution is detected in the amygdala and the anterior cingulate, auditory and dorsolateral prefrontal cortices, with an inverse relationship between unconditioned stimulus expectation and unconditioned response. ${ }^{19}$ Computer simulation showed that activity in the amygdala and anterior cingulate cortex is related to unconditioned stimulus predictability, while activity in the dorsolateral prefrontal cortex and insula is accounted for by attention-modulated representation of conditioned stimuli. ${ }^{19}$

In general, higher forebrain areas are involved in the response to early threat, including assignment of a stimulus to the fear domain and fear control, whereas 'automatically' perceived, imminent danger induces rapid downstream reactions from midbrain nuclei, such as the amygdala. ${ }^{20}$ The functions of the amygdala are summarised in Table I.

\section{Fear learning}

Fear learning is a rapid and persistent process that promotes defence against threats and reduces the need to relearn about danger. The lateral prefrontal cortex may diminish fear by decreasing activity in the amygdala similarly to the ventromedial prefrontal cortex, which inhibits the amygdala during extinction. ${ }^{49}$ The hippocampus and the amygdala are involved during late phases of extinction, and are therefore important for maintaining extinction. ${ }^{50,51}$

When it comes to fear, flexibility is important. Fear behaviour should adjust when circumstances change. A central yet neglected aspect of fear modulation concerns the ability to change fear responses if a once-threatening stimulus becomes safe, or a once-safe stimulus becomes threatening. In these situations, which require accurate responses under continuous stress, the inhibition of fear and the development of fear reactions co-occur, but are directed at different targets. Using functional neuro-imaging in conjunction with a fear-conditioning reversal paradigm, Schiller et al. ${ }^{12}$ showed safe stimuli that previously predicted danger and 'naive' safe stimuli to dissociate within the ventromedial

Table I. Amygdala activation in response to fear

\begin{tabular}{lll}
\hline & Major activation & Minor activation \\
\hline Stimulus type & Emotional stimuli & Neutral stimuli \\
Emotion type & Negative & Happiness and positive \\
Sensory type & Gustatory-olfactory and visual & Internal stimuli \\
Attention & Passive processing & Active task instructions \\
Behavioural relevance & Letter stimuli, & Letter stimuli \\
Response to faces & behaviourally relevant & with less behavioural relevance \\
Response to fearful faces & Fearful & Neutral \\
& Fear expressed by members of the same & Fear expressed by members of another \\
& cultural group & cultural group \\
\hline
\end{tabular}


prefrontal cortex, which is more engaged by the former. Fearpredictive stimuli are tracked by the amygdala and the striatum, which flexibly shift their responses from one stimulus to another. Prediction errors associated with reversal learning correlate with striatal activation. All this shows brain flexibility in fear control and its readjustment in tracing the valence of environmental change. ${ }^{12}$

Delgado et al. ${ }^{49}$ suggested that humans may have developed complex cognitions that can aid in regulating emotional responses while at the same time utilising phylogenetically shared mechanisms of extinction when dealing with stimuli with an immediate 'die or survive' response to threat. It seems that the cortex, based on its previous statistics, processes the likelihood of certain outcomes when confronted with certain threatening stimuli. It further seems that, when the outcome is not immediately negative and other factors (such as context) are taken into account, the result of this processing may involve a 'wait-and-see' approach. This may explain why some people may experience pleasure while watching thriller (or even horror) movies. Brain areas involved in fear learning are shown in Table II. A model showing brain areas involved in emotional processing is shown in Fig. 2.

\section{Conclusions}

Several studies have targeted brain regions involved in human fear. The most important finding is the central role of the amygdala and a subcortical network that interacts with the prefrontal cortex to modulate the fear response in order to cope more effectively with environmental and social demands

In the future such studies may help in the identification of more effective treatment approaches. Improvements in functional neuro-imaging and other imaging techniques may contribute to greater clarification of the neurocircuitry of fear perception and fear response. Such improved imaging techniques may also assist in assessment of the responses to both drug treatment and psychotherapy. It will be of particular interest to investigate the area

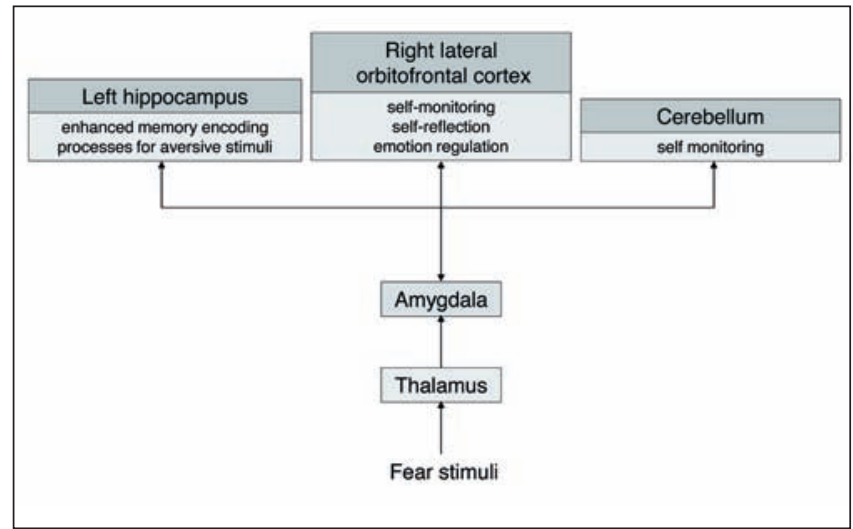

Fig. 2. Main brain areas that modulate the emotional processing of fear stimuli.

at the boundary between the anxiety disorders and the normal fear response. An important step for future research will be the integration of functional imaging studies with neurophysiological, neuropsychological and behavioural ones.

\section{References}

1. Ohman A. The role of the amygdala in human fear: automatic detection of threat. Psychoneuroendocrinology 2005;30:953-958.

2. Aggleton JP. The Amygdala: Neurobiological Aspects of Emotions, Memory and Mental Dysfunction. New York: John Wiley \& Sons, 1992

3. LeDoux JE. The Emotional Brain: The Mysterious Underpinnings of Emotional Life. New York: Simon \& Schuster, 1996

4. Davis M. Neural circuitry of anxiety and stress disorders. In: Davis KL, Charney D, Coyle JT, Nemeroff C, eds. Neuropsychopharmacology. The Fifth Generation of Progress. Philadelphia, PA: Lippincott Williams \& Wilkins, 2002: $931-951$

5. Davis M. Neurobiology of fear responses: the role of the amygdala. J Neuropsychiatry Clin Neurosci 1997;9:382-402

6. Amaral DG, Behniea H, Kelly JL. Topographic organization of projections from the amygdala to the visual cortex in the macaque monkey. Neuroscience 2003; 1 18:1099-1120.

7. Milad MR, Quirk GJ, Pitman RK, Orr SP, Fischl B, Rauch SL. A role for the human dorsal anterior cingulate cortex in fear expression. Biol Psychiatry 2007;62:11911194

8. Morgan MA, LeDoux JE. Differential contribution of dorsal and ventral medial prefrontal cortex to the acquisition and extinction of conditioned fear in rats. Behav Neurosci 1995; 109:681-688

Table II. Brain areas involved in fear learning processes

\begin{tabular}{|c|c|}
\hline Area & Function \\
\hline Lateral prefrontal cortex regions & Cognitive emotion regulation strategies \\
\hline Ventromedial prefrontal cortex & $\begin{array}{l}\text { Inhibits the amygdala during extinction } \\
\text { Retention of extinction }\end{array}$ \\
\hline Hippocampus & Gustatory-olfactory and visual \\
\hline Striatum & Flexibly shifts fear responses from one stimulus to another \\
\hline Insula & \multirow{5}{*}{ Anticipation of aversive affective images } \\
\hline Somatosensory cortex & \\
\hline Parahippocampal gyrus & \\
\hline Anterior cingulate cortex & \\
\hline Dorsolateral prefrontal cortex & \\
\hline
\end{tabular}


9. Sanders MJ, Wiltgen BJ, Fanselow MS. The place of the hippocampus in fear conditioning. Eur J Pharmacol 2003;463:217-223.

10. Alvarez RP, Biggs A, Chen G, Pine DS, Grillon C. Contextual fear conditioning in humans: cortical-hippocampal and amygdala contributions. J Neurosci 2008;28:6211-6219.

11. Sehlmeyer C, Schöning S, Zwitserlood P, et al. Human fear conditioning and extinction in neuroimaging: a systematic review. PLoS One 2009;4:e5865.

12. Schiller $D$, Levy I, Niv Y, LeDoux JE, Phelps EA. From fear to safety and back: reversal of fear in the human brain. J Neurosci 2008:28:11517-11525.

13. LaBar KS, Gatenby JC, Gore JC, LeDoux JE, Phelps EA. Human amygdala activation during conditioned fear acquisition and extinction: a mixed-trial fMRI study. Neuron 1998;20:937-945

14. Phan KL, Wager T, Taylor SF, Liberzon I. Functional neuroanatomy of emotion: a metaanalysis of emotion activation studies in PET and fMRI. Neurolmage 2002;16:331348.

15. Garrett AS, Maddock RJ. Separating subjective emotion from the perception of emotion-inducing stimuli: an fMRI study. Neurolmage 2006;33:263-274.

16. Derntl B, Habel U, Windischberger $C$, et al. General and specific responsivenes of the amygdala during explicit emotion recognition in females and males. BMC Neurosci 2009; 10:91

17. Pichon S, de Gelder B, Grezes J. Two different faces of threat. Comparing the neural systems for recognizing fear and anger in dynamic body expressions. Neurolmage 2009;47:1873-1883

18. Etkin A, Wager TD. Functional neuroimaging of anxiety: a meta-analysis of emotional processing in PTSD, social anxiety disorder, and specific phobia. Am J Psychiatry 2007; 164:1476-1488.

19. Dunsmoor J, Schmajuk N. Interpreting patterns of brain activation in human fear conditioning with an attentional-associative learning model. Behav Neurosci 2009:123:851-855.

20. Mobbs D, Marchant JL, Hassabis D, et al. From threat to fear: the neural organization of defensive fear systems in humans. J Neurosci 2009;29:1 2236-1 2243

21. Hariri AR, Bookheimer SY, Mazziotta JC. Modulating emotional responses: effects of a neocortical network on the limbic system. Neuroreport 2000; 1 1:43-48

22. Hariri AR, Mattay VS, Tessitore A, Fera F, Weinberger DR. Neocortical modulation of the amygdala response to fearful stimuli. Biol Psychiatry 2003;53:494-50 1

23. Taylor SF, Phan KL, Decker LR, Liberzon I. Subjective rating of emotionally salient stimuli modulates neural activity. Neurolmage 2003; 18:650-659

24. Gotffried JA, Dolan RJ. Human orbitofrontal cortex mediates extinction learning while accessing conditioned representations of value. Nat Neurosci 2004;7:1 144-1 152.

25. Freeman JHJ, Cuppernell C, Flannery K, Gabriel M. Limbic thalamic, cingulate cortical and hippocampal neuronal correlates of discriminative approach learning in rabbits. Behav Brain Res 1996;80:123-136.

26. Buchanan SL, Powell DA. Cingulate cortex: its role in Pavlovian conditioning. J Comp Physiol Psychol 1982;96:755-774.

27. Liang X, Zebrowitz LA, Aharon I. Effective connectivity between amygdala and orbitofrontal cortex differentiates the perception of facial expressions. Soc Neurosci 2009;4: 185-196.

28. Ward R, Calder AJ, Parker M, Arend I. Emotion recognition following human pulvinar damage. Neuropsychologia 2007; 45:1973-1978.

29. Anderson AK, Phelps EA. Is the human amygdala critical for the subjective experience of emotion? Evidence of intact dispositional affect in patients with amygdala lesions. Cogn Neurosci 2002; 14:709-720
30. Steele JD, Lawrie SM. Segregation of cognitive and emotional function in the prefrontal cortex: a stereotactic meta-analysis. Neurolmage 2004;21:868-875.

31. Lieberman MD, Jarcho JM, Berman S, et al. The neural correlates of placebo effects: a disruption account. Neurolmage 2004;22:447-455.

32. Simmons A, Matthews SC, Stein MB, Paulus MP. Anticipation of emotionally aversive visual stimuli activates right insula. Neuroreport 2004; 15:2261-2265.

33. Yágüez L, Coen S, Gregory LJ, et al. Brain response to visceral aversive conditioning: a functional magnetic resonance imaging study. Gastroenterology 2005; 128:18191829

34. Costafreda SG, Brammer MJ, David AS, Fu CHY. Predictors of amygdala activation during the processing of emotional stimuli: a meta-analysis of 385 PET and FMRI studies. Brain Res Rev 2008:58:57-70.

35. Hadjikhani N, Hoge R, Snyder J, de Gelder B. Pointing with the eyes: the role of gaze in communicating danger. Brain Cogn 2008;68:1-8.

36. Gamer $M$, Büchel $C$. Amygdala activation predicts gaze toward fearful eyes. J Neurosci 2009:29:9123-9126.

37. Hardee JE, Thompson JC, Puce A. The left amygdala knows fear: laterality in the amygdala response to fearful eyes. Soc Cogn Affect Neurosci 2008;3:47-54.

38. Das P, Kemp AH, Liddell BJ, et al. Pathways for fear perception: modulation of amygdala activity by thalamo-cortical systems. Neurolmage 2005;26:141-148.

39. Siman-Tov T, Papo D, Gadoth N, et al. Mind your left: spatial bias in subcortical fear processing. J Cogn Neurosci 2009;21:1782-1789.

40. Asghar AUR, Chiu YC, Hallam G, et al. An amygdala response to fearful faces with covered eyes. Neuropsychologia 2008;46:2364-2370.

41. Carlson JM, Reinke KS, Habib R. A left amygdala mediated network for rapid orienting to masked fearful faces. Neuropsychologia 2009;47:1386-1389.

42. Ousdal OT, Jensen J, Server A, Hariri AR, Nakstad PH, Andreassen OA. The human amygdala is involved in general behavioural relevance detection: evidence from an event-related functional magnetic resonance imaging Go-NoGo task. Neuroscience 2008; 156:450-455

43. Chiao JY, lidaka T, Gordon $\mathrm{HL}$, ef al. Cultural specificity in amygdala response to fear faces. J Cogn Neurosci 2008;20:2167-2174.

44. Haruno M, Frith CD. Activity in the amygdala elicited by unfair divisions predicts social value orientation. Nat Neurosci 2010;13:160-161.

45. Aragona M, Puzella A. Come cambia l'empatia per il dolore nelle neuroscienze: influenze reciproche tra piano concettuale e sperimentazione. Giornale Italiano d Psicopatologia 2010;16:309-320

46. Williams LM, Das P, Liddell BJ, Kemp AH, Rennie CJ, Gordon E. Mode of functional connectivity in amygdala pathways dissociates level of awareness for signals of fear. J Neurosci 2006;26:9264-9271.

47. Deeley $Q$, Daly EM, Azuma $R$, et al. Changes in male brain responses to emotional faces from adolescence to middle age. Neurolmage 2008;40:389-397.

48. Root JC, Tuescher $O$, Cunningham-Bussel A, et al. Frontolimbic function and cortisol reactivity in response to emotional stimuli. Neuroreport 2009;20:429-434.

49. Delgado MR, Nearing KI, Ledoux JE, Phelps EA. Neural circuitry underlying the regulation of conditioned fear and its relation to extinction. Neuron 2008;59:829838.

50. Phelps EA, Delgado MR, Nearing KI, LeDoux JE. Extinction learning in humans: role of the amygdala and vmPFC. Neuron 2004;43:897-905.

51. Milad MR, Wright Cl, Orr SP, Pitman RK, Quirk GJ, Rauch SL. Recall of fear extinction in humans activates the ventromedial prefrontal cortex and hippocampus in concert. Biol Psychiatry 2007;62:446-454. 